\title{
LA APERTURA DEL PSICOANÁLISIS A LA REALIDAD SOCIAL Y PERSONAL DE LOS HOMBRES Y DE LAS MUJERES ${ }^{1}$
}

\author{
Joan Coderch de Sans ${ }^{2}$ \\ SEP (IPA), IPR \\ Barcelona
}

Como consecuencia del formidable avance del paradigma relacional en el campo del psicoanálisis a partir de la última década del pasado siglo, se impone la convicción -fundamentada tanto en la experiencia clínica como en las aportaciones empíricamente comprobadas de la biología y las ciencias neurológicas- de que la mente humana se construye a través de la interacción del bebé con la madre y con aquellos que constituyen la matriz socio/relacional en la cual nace y evoluciona. A partir de esta perspectiva, el pensamiento psicoanalítico se despliega en dos direcciones. Por un lado, surge el interés por el estudio y la comprensión de la sociedad de la cual el mismo psicoanálisis forma parte y en el seno de la cual crece y se desarrolla o declina y cae. Por otro, en la práctica clínica del psicoanálisis se impone la imperiosa necesidad de dejar de centrar la atención del analista exclusivamente en la situación analítica y la transferencia para dirigir la mirada también hacia la vida cotidiana de los pacientes, tanto en la dimensión social como en la interpersonal, en la que se repiten los patrones procedimentalmente organizados de respuesta a las situaciones que surgen en el análisis y en la realidad externa. Cuando estos patrones son perturbadores y desadaptados como resultado de las inadecuadas relaciones emocionales en la infancia del sujeto, pueden ser modificados gracias a la sintonización psicobiológica paciente-analista que tiene lugar en la interacción entre ambos.

Palabras clave: Psicoanálisis Relacional, Interacción terapéutica, Realidad Social, Vida personal.

As a result of the formidable progress or the relational paradigm in the field of psychoanalysis that has been taking place from the last decade of the former century, it must be accepted -an acceptance based as much on clinical experience as on empirically proved contributions from biology and neurological sciences- that human mind is formed through interaction of the new-born with the mother and with those that form the sociorelational matrix in which the child is born and evolves. From this perspective on, the psychoanalytical thought is deployed into two directions. On the one side, there is an upsurge in the interest for studying and understanding of the society of which psychoanalysis itself is a part and inside which it grows and develops or it declines and falls. On the other side, in the practice of psychoanalysis it gets absolutely essential to stop centering the analyst's attention exclusively on the analytical situation and transference in order to behold also the patient's daily life, both in the social and in the interpersonal dimensions, in which standards procedimentally organized for answering at situations that appear during analysis and in external reality are often repeated. When these standards turn up to be disturbing and maladapted as a result of the inadequate emotional relationships during the patient's childhood they can be modified thanks to a psychobiological tuning patient-analyst that takes place in the interaction between both.

Key Words: Relational Psychoanalysis, Therapeutic Interaction, Social Reality, Personal Life. English Title: The opening of Psychoanalysis to the social and personal reality of men and women.

\section{Cita bibliográfica / Reference citation:}

Coderch, J. (2018). La apertura del psicoanálisis a la realidad social y personal de los hombres y de las mujeres. Clínica e Investigación Relacional, 12 (1): 11-28. [ISSN 1988-2939] [Recuperado de www.ceir.info ] DOI: 10.21110/19882939.2018.120101 


\section{Perspectiva general de la apertura del psicoanálisis a la realidad social.}

Lo primero que deseo decir es que las dos magníficas y estimulantes Ponencias que nos han sido presentadas, Comunidad, a cargo de Andrew Samuels, y Pareja, a cargo de Phil Ringstrom, merecían un aplauso, por su novedad y atrevimiento, ya antes de ser leídas y escuchadas, y son acreedoras de los más rendidos plácemes después de serlo, como también son dignos de ellos los discutidores L. Raimundo Guerra y Neri Daurella3. Porque en todo su conjunto, ateniéndonos al lema general de estas Jornadas, ha quedado claro que se han venido abajo las barreras que durante tantos años han mantenido al psicoanálisis encerrado en la torre de marfil de un mundo intrapsíquico sin relación alguna con la realidad externa a él.

Nos hacen ver los Ponentes, los Discutidores y todos aquellos que han intervenido en el debate, de una u otra forma, que el psicoanálisis relacional no sólo ha entrado de lleno en el diálogo con la realidad social en la que vivimos sino que, además, creo que todos podemos ahora aceptar plenamente que tal realidad está inserta de lleno en la misma situación clínica en la que siempre hemos trabajado, porque el clásico "aquí y ahora", como una pura situación entre dos personas, sin interferencia de ninguna clase, es una simple entelequia, puesto que paciente y analista llevan a sus espaldas todos los contextos en los han vivido y viven, cosa que se pone de relieve en todos y cada uno de los momentos de encuentro de la díada analítica, porque no se sale de un contexto y se entra en otro abandonado el primero como se va de una habitación a otra. La apertura a la realidad de la vida que han puesto en evidencia los Ponentes, como todos los que han intervenido en el debate, está sacudiendo ya todo el edificio psicoanalítico, desde sus fundamentos teóricos y conceptuales hasta los más delicados y sutiles matices de su práctica clínica, y a algunos, sólo a algunos, de estos cambios y positivos avances me referiré, de ahora en adelante, en mi exposición.

Lo visto en estas Jornadas nos lleva a la articulación de la situación analítica que viven paciente y analista con aquello que se halla más allá de sus límites y, por tanto, con la sociedad y con el transcurrir de los pacientes en su seno. Así, el psicoanálisis contemporáneo deja de centrarse, tan sólo, en las dimensiones inconscientes de la mente de los pacientes, para hacerlo en la totalidad de la misma y en su plena inserción en la sociedad en la que viven. Aunque, ciertamente, distintos tipos de actos psíquicos, activan y ponen en marcha diferentes zonas, corticales y subcorticales del cerebro, el estudio del mismo mediante las técnicas de imagen pone de manifiesto que, en cualquiera de ellos, es todo el cerebro el que se manifiesta, e, igualmente, que en todo acto psíquico interviene, de una u otra forma, la totalidad de la mente $y$, por tanto, de los patrones o principios de organización de respuesta 
con los que el sujeto reacciona a todas las situaciones corporales, intrapsíquicas o interpersonales que la vida le va presentando (Kandel, E., Schwartz,J., y Jesell, T., 2001; Kandel, E., 2005).

Debemos tener en cuenta, así mismo, que la situación analítica y la realidad social no son dos sistemas separados que pueden interaccionar entre sí, sino que las cosas están mucho más enmarañadas. A la vez que cabe distinguir estos dos sistemas, no debemos olvidar que cada uno de ellos es un elemento del otro sistema, porque la comunidad psicoanalítica y el psicoanálisis como terapéutica forman ambos parte de la realidad cultural y social, y ésta, a su vez, forma parte de la práctica clínica, dado que paciente y analista viven, luchan, padecen y gozan en ella, y en ella se manifiesta la salud o la patología de uno y otro, dado que todos los procesos psíquicos son relacionales y, como consecuencia, la evolución del psicoanálisis ha sido siempre paralela a la evolución y cambios de la sociedad en la que se desenvuelve y crece, o declina y cae.

No me cabe duda de que, en estos momentos, los psicoanalistas han de centrar su atención en la comunidad y la sociedad en la que viven los pacientes por una razón ante la que no podemos permanecer ciegos por más tiempo, la de que, en distintos grados y tonalidades, el sufrimiento de muchos seres humanos en el momento presente se encuentra provocado muy directamente por las características propias y formas de organización de la actual realidad social, porque se trata de una sociedad en crisis, desvinculada, carente de valores que den sentido a una vida caótica, judicializada y que vive, además, bajo la amenaza del terror (Tizón, J., 2010, 2015; Coderch, J. 2016b; Coderch, J. y Plaza, A., 2016). En este sentido, quiero señalar, de manera muy breve, que el psicoanálisis, ha pasado por cuatro etapas en su forma de entender los factores y variables que intervienen en el origen del sufrimiento psíquico que ha empujado a los pacientes a solicitar la ayuda de los analistas. En la primera etapa, los trastornos emocionales que presentaban las pacientes de Freud fueron atribuidos, de acuerdo con la teoría traumática, nunca del todo abandonada (Codosero, A., 2012), a los abusos sexuales sufridos en la infancia. En la segunda, la etiología de los trastornos emocionales se refirió al conflicto intrapsíquico de estirpe edípica, endógeno y sin relación alguno con la realidad de la vida de los pacientes. En la tercera etapa, ya propia del paradigma relacional, tal sufrimiento ha sido juzgado como la consecuencia de una insatisfacción grave de las necesidades emocionales de amor, cuidado, comunicación y correspondencia vivida por los pacientes en su infancia. $Y$ yo pienso que, actualmente, estamos en una cuarta etapa en la que, junto y de la mano con la tercera, se nos hace evidente que la civilización deshumanizada y tecnocrática, la confusión en cuanto a los valores a los que debemos atenernos, la incertidumbre en cuanto al porvenir, el todos contra todos y la creciente individualización son las más significativas variables que propician el incremento progresivo de los trastornos 
emocionales. (Baladier, G, 1988; Barcellona, P.,1992; Lyotard, J.F.., 1984; Sennet, R.,1998; Souza, Ma.L.,1999; Bauman, Z., 2000, 2001; Lipovetsky, G., 2004; Coderch, ,2016b; Coderch, J, Castaño, R., Codosero, A., Daurella, N. y Rodríguez-Sutil, C., 2014; Coderch, J. y Plaza, A., 2016). Y estas circunstancias dan lugar a que, en gran número de casos, personas a quienes podemos considerar portadoras de una razonable salud mental y que, hasta un momento determinado de su vida, habían sido capaces de trabajar, amar y ser amadas se derrumban y rompen, hundidas por la enorme presión de uno o varios de los contextos en los que viven. Es decir, la caótica y desorganizada sociedad en la que se halla el mundo que llamamos civilizado es la que, sin lugar a dudas, provoca la aparición de perturbaciones emocionales en gran número de hombres y mujeres en nuestra época.

Todos, analistas y pacientes vivimos en tal mundo y sería ingenuo y absurdo tratar de describirlo ahora en su totalidad. Pero la investigadora social $\mathrm{M}^{\mathrm{a}}$ Lourdes de Souza, en su trabajo sobre la individualidad postmoderna (1999), al citar una frase de Pietro Barcellona- el insigne investigador italiano de la postmodernidad tras la caída del Muro de Berlín, en las últimas décadas del pasado siglo- resume con pasmosa claridad la situación actual. Afirma de Souza que el autismo de la era individualista se ha convertido, como expresó Barcellona: en una extraña Torre de Babel en la que todo el mundo consigue conectar con la red informática pero ya no logra hablar con el vecino de enfrente". Esta frase fue escrita por Barcellona en 1990, y me parece difícil no admitir que el individualismo, la incomunicación y la legalización normativa de las relaciones sociales e incluso interpersonales, que han substituido a la confianza, a la amistad, a la compasión y al amor se han incrementado exponencialmente desde entonces (Coderch, J. (2016b). No es de extrañar que muchos de entre quienes viven en este tipo de civilización sufran alteraciones emocionales, porque sabemos que todo proceso psíquico es relacional, y si falla la relación con el otro y, por añadidura, con la Comunidad que todo lo envuelve, falla la mente. En similar sentido, Barcellona, con gran realismo se lamenta en su libro Postmodernidad y Comunidad (1992):

Poco importa que las grandes concentraciones del llamado tercer capitalismo estén desintegrando la soberanía de los Estados y la libertad de información, o entregando áreas geográficas enteras a poderes criminales. Lo que cuenta es solo esa gran simplificación de la proclamada complejidad moderna en el paradigma universal de la "mercancía" y del "dinero" (p.9).

Como podemos ver a través de estas palabras, la visión de la actual sociedad capitalista por parte de Barcellona era francamente pesimista y ofrece pocas esperanzas.

Nos señala De Souza la manera lamentable de como la reducción de la vida social a las leyes y formas jurídicas lleva a la postergación y disolución de la individualidad y de las relaciones expresa. Este material es para uso científico y profesional exclusivamente y puede contener información clínica sensible. Los editores no se responsabilizan de los contenidos de los autores. Dirigir las consultas sobre derechos y autorizaciones a ceir@psicoterapiarelacional.es 
interpersonales en la experiencia jurídica, cosa que conduce a que tales relaciones sean "formalizadas"a través de normas y leyes (p.324.). Yo creo que todos tenemos la experiencia de que la confianza entre las personas ha sufrido una alarmante disminución, y en muchos de nuestros pacientes hemos podido observar, subyacente a la ansiedad crónica, un vivir cautelosamente, mirando a cada lado, como el que anda en una selva rodeada de peligros, siempre atento a los riesgos que pueden presentarse.

Ambos autores, Barcellona y de Souza, nos hablan, entre otras consideraciones y reflexiones socioeconómicas y políticas, de la necesidad, para remediar la crisis actual de la sociedad, de devolver un "sentido"de la vida a cada individuo, vinculando su autonomía con la comunidad. Es decir, reforzar la autonomía de cada individuo, de sus particularidades y diferencias, interconectándola con las razones de la Comunidad, con el carácter esencialmente social del proceso de formación de la individualidad (de Souza, p.335).

En tanto que psicoanalista pienso que, con estas palabras, con las que me siento identificado, se nos señala una actitud muy parecida a la que sabemos imprescidible para la formación del self: el reconocimiento del objeto (del otro), como igual a mí, pero distinto y separado, con cuyo recíproco reconocimiento puedo percibir mi propio self, igual al otro y diferente a él. Es decir, la vinculación con el otro refuerza mi self y perfila y destaca mi autonomía, y este conocimiento psicoanalítico nos conduce a comprender, en el campo social, las imprescindibles vinculaciones y el reconocimiento entre cada individuo en particular y la Comunidad de la que forma parte, para que tanto esta última como él mismo gocen de una buena salud mental.

Ahora bien, muchos hombres y mujeres han buscado y encontrado otro tipo de respuesta para protegerse del malestar social en el que nos encontramos, de la confusión de valores, de la diversidad de morales, del individualismo, de la falta de vínculos sociales y del sentimiento de soledad que de ello se deriva, y esta búsqueda ha dado lugar a la formación de grupos comunitarios locales, lo que se ha denominado comunitarismo, cuyo más destacado campeón parece ser el filósofo Alasdair Maclntyre, a quien una de sus prologuistas, la profesora de Ética de la Universidad de Barcelona Victoria Camps, Ilama neo-aristotélico. MacIntyre, en su libro Tras la Virtud (1987), se muestra muy pesimista respecto a la posibilidad de llevar a cabo y lograr en la práctica, la Universalidad de los Derechos Humanos, pero apunta otra esperanza, y en el último párrafo del libro, que no cito en su totalidad para no alárgame, y como conclusión afirma:

Lo que importa ahora es la construcción de formas locales de comunidad, dentro de las cuales la civilidad, la vida moral y la vida intelectual puedan sostenerse a través de las nuevas edades obscuras que caen ya sobre nosotros. Y si la tradición de las virtudes fue capaz de sobrevivir a las expresa. Este material es para uso científico y profesional exclusivamente y puede contener información clínica sensible. Los editores no se responsabilizan de los contenidos de los autores. Dirigir las consultas sobre derechos y autorizaciones a ceir@psicoterapiarelacional.es 
edades obscuras, no estamos enteramente faltos de esperanza... No estamos esperando a Godot, sino a otro sin duda muy diferente, San Benito. (p.322).

Por cierto, que Z. Bauman (2000) se muestra más bien anti comunitarista y afirma que las comunidades locales son una postulación, un proyecto y no una realidad, algo que viene después y no antes de la elección individual. Rechaza, de manera tajante, la tendencia marcadamente normativa y reglamentada que se observa en algunas formas de comunitarismo, bajo pretexto de la búsqueda de una identidad supuestamente esencialista e inmutable que todos los miembros deben compartir.

Desde la perspectiva de Maclntyre, el sociólogo Victor Turner (1988), evidentemente refiriéndose a las comunidades locales, no a la gran Comunidad que formamos todos sin distinción de creencias y valores, así lo expresa:

Comunidad es una relación entre individuos concretos, históricos y con una idiosincrasia determinada, que no se encuentran divididos en roles y estatus, sino puestos los unos frente a los otros, un poco a la manera del yo y el tú del que habla Martin Buber (p. 102).

Queda claro que este autor piensa en una forma de comunitarismo en la que no existe ninguna clase de regulación para ejercer el control social de sus miembros, ni estructuras de poder, sino una autoridad colectiva destinada a estimular el crecimiento moral de sus miembros. Pone, como ejemplo, las primeras comunidades franciscanas.

No parece extraño que muchos seres humanos, desorientados, invadidos por un sentimiento de soledad y aturdidos por la gran cantidad de valores contradictorios y de dispares propuestas que lanzan los políticos y líderes sociales, busquen refugio en el agrupamiento con otros seres humanos, con otros semejantes que sienten persiguen la misma meta y formen comunidades, a veces muy numerosas y otras más reducidas en las que se comparte la vivienda. Pero la experiencia muestra que las cosas nunca son tan fáciles. A lo largo de mi vida profesional he tratado a varios pacientes adscritos a alguna forma de comunidad local de diversa índole, y puedo asegurar que los problemas, dificultades inesperadas y contradicciones que surgen son desalentadores y provocan que muchos de los seres humanos que se han acogido a ellas sientan roto su compromiso y se encuentren a sí mismos en un terrible estado de soledad y de desorientación social (Lyotard.J.F., 1984; Bauman, Z., 2000; Ávila-Espada, A., 2015, 2016b). Como podemos ver, pues, la elección entre Comunidad y comunitarismo es a gusto, o tal vez a disgusto, de cada uno.

En cuanto a la cuestión de las relaciones de pareja y de la sexualidad, ésta se halla más cercana a los psicoanalistas que el de la Comunidad, porque se presenta, de una u otra manera, en el trabajo diario de los psicoanalistas, y el interés que ahora se despierta por la realidad de 
la existencia de los pacientes desvela la enorme importancia de esta variable en el estado psíquico de los mismos. Por ello, deseo hablar ahora, brevemente, acerca de la cada vez más frecuente ruptura de los matrimonios y de las parejas establecidas con propósito de larga duración, desde la perspectiva de la apertura al diálogo con la antropología (Duch, L., y Mèlich, J.C., 2004; Castaño, R., 2011; Guerra-Cid, R., 2013). Es propio de nuestra individualista y competitiva sociedad que hombres y mujeres busquen, por todos los medios, el desarrollo de sus deseos y proyectos de vida, de su autonomía y de su afirmación, y, para ello, se afanan en proveerse de todos los recursos y disponibilidades para satisfacerlos, de manera egocéntrica y enfrentada a los otros. Esto hace que, actualmente, la elección de pareja se realiza, por parte de gran número de personas, no según la búsqueda de alguien valioso y admirable por sus cualidades en tanto que ser humano, de alguien que por su comportamiento y manera de ser se hace digno de ser amado o amada, sino que más bien se emprende tal elección desde una perspectiva funcional e instrumental, tomando como punto de mira elegir, como pareja, a alguien cuyas capacidades, recursos o pertenencia a determinado status familiar, económico o profesional puede ayudar a llevar a cabo y alcanzar los propios programas y las metas deseadas. Es decir, se elige la pareja como instrumento útil para los logros personales, o para la mera satisfacción sexual. No es de extrañar que gran parte de las parejas formadas bajo estas premisas no aguanten los múltiples cambios y variadas circunstancias que van presentándose y se rompan, más tarde o más temprano.

Distintamente, la relación de pareja fundada sobre la base del respeto a los valores humanos de la pareja elegida y en el amor, éste- que no debe ser confundido con el enamoramiento- conduce a la profundización en la intersubjetividad, dirigida a desvelar, reconocer y poner más a la luz del día aquello que cada uno de los miembros ama en el otro u otra, a la vez que a despertar recursos y posibilidades, en él o ella, que estaban como adormecidos, y, cuando las cosas son así, el amor obra como un manantial de vida que estimula y favorece, en ambos componentes, el acrecentamiento y mayor desarrollo de las cualidades y valores que, recíprocamente, les han embelesado y atraído. Así, la intersubjetividad creada entre los que se aman conduce a un enriquecimiento mutuo que puede continuar durante el resto de la vida (Summers, F., 2015).

\section{Consecuencias de la apertura a la realidad en la clínica psicoanalítica.}

De lo que se trataré ahora, pues, es de tomar consciencia de los formidables avances que ha promovido en la clínica psicoanalítica esta apertura a la totalidad de la mente y de la vida de los seres humanos, así como al entorno social que nos rodea a todos. Tal apertura conduce, como es de esperar, a la articulación de la situación analítica con el mundo de la existencia de 
los pacientes, en una cíclica oscilación del interés del trabajo analítico centrado ya sea en el aquí y ahora de la sesión, ya sea en su vida cotidiana (Wachtel, P.L. 2012). Pero no solamente esto, sino que el dirigir la mirada hacia el exterior nos permite dejar de ver a los pacientes tan sólo como los portadores de un inconsciente reprimido que debemos interpretar. A mi juicio, tanto la experiencia clínica como las aportaciones interdisciplinares nos han enseñado la necesidad de que el analista no se ciña únicamente a aquello que sucede en la situación analítica, a lo que habitualmente llamamos la transferencia y la contratransferencia, sino que su indagación debe abarcar, también, lo que se presenta en la realidad de la vida de los pacientes. Y ello debe ser así, porque las alteraciones emocionales, la ansiedad, las respuestas repetitivas y los patrones organizadores perturbadores y desadaptados frente a las diversas situaciones a las que la existencia enfrenta a todos los seres humanos surgen, de continuo, en las relaciones interpersonales que los pacientes mantienen con quienes forman sus distintos contextos, y, lo que es más, estas experiencias del presente también influyen y modifican las más primitivas experiencias traumáticas que vivió cada paciente y que se hallan en el origen de sus sufrimientos, de sus conflictos consigo mismo, con los otros y de su demanda de ayuda $y$, por tanto, nos ha de quedar claro que los patrones de respuesta originados en tales primitivas experiencias no permanecen congelados e inmutables ante las nuevas relaciones propias de la edad adulta, sino que se modifican y despliegan en el calor de la vida.

En estos momentos, gracias a la apertura a la realidad externa en la propia situación analítica, podemos entender que el paciente dice lo que dice, que sus palabras no únicamente son la expresión enmascarada de un mundo intrapsíquico inconsciente, oculto, no susceptible de ser comunicado verbalmente, sino que, con ellas, los pacientes tratan de que el analista tome parte, con su comprensión y empatía, en lo bueno y lo malo de su existencia en el mundo, en sus deseos, en sus esperanzas, en sus frustraciones y en sus logros. La comunicación verbal y subverbal de los pacientes no hemos de entenderla, de ninguna manera, como una mera capa, el contenido manifiesto, que esconde el verdadero sentido de su expresión, el contenido latente que el analista ha de interpretar a tenor de las teorías que sostiene. No, no debemos conformamos con estas rudimentarias divisiones de la comunicación, por un lado, y de la misión del analista, por el otro (Coderch, J., 2015, 2016a; Ávila-Espada, A., 2016a).

Esta apertura a la realidad de la vida, rompiendo los rígidos esquemas y normas que han prevalecido en el psicoanálisis durante casi un siglo, también ha alcanzado al analista y a sus funciones, y nos empuja a abandonar la regla de la distancia analítica y la idealizada figura del analista "suficientemente analizado", según la típica adjetivación, imperturbable y frio, enteramente dueño de sus reacciones emocionales y únicamente centrado en su labor interpretativa, y se nos ha hecho evidente la necesidad de que, muy al contrario, acompañe a 
sus pacientes en sus emociones, sufrimientos y esperanzas y sea capaz de sintonizar psicobiológicamente con ellos, algo de lo que volveré a hablar más adelante, y les ame con amor terapéutico en tanto que seres humanos. Personalmente, pienso que el espíritu que ha de regir la relación con los pacientes queda anunciado claramente por Ferenczi con sus palabras: Sin Simpatía no hay Curación (1932).Y también en las palabras de Donna Orange (2006), cuando nos dice que nuestra actitud de acogimiento, hospitalidad y compasión debe expresar a cada paciente: your suffering is human suffering, and when the bell tolls for you, it also tolls for me (tu sufrimiento es sufrimiento humano, y cuando las campanas doblan por ti también doblan por mí, p.15). Este espíritu, que ofrece generosa seguridad y compañía, es el que impulsa a los paciente a correr el riesgo, prescindiendo de sus medidas defensivas para evitar la retraumatización, de vivir su interacción con el analista no exclusivamente a través del lenguaje verbal y subverbal, sino de vivirla en toda su plenitud a través de los enactments (Bromberg, P, 2011), es decir, mediante el despliegue y escenificación de los estados disociados del self, aquellos estados que no pudieron ser simbolizados debido a la situación afectivamente traumática en la que fueron vividos. Y ello también conduce al analista a no cortar la puesta en escena del sufrimiento del paciente con racionalizadas interpretaciones, sino a mantener una actitud de introspección empática para observar la posible aparición, en su propia experiencia subjetiva, de las emociones, fantasías y estados de ánimo paralelos y correspondientes a los del paciente, así como al despliegue de sus propios enactments, para que, en el interjuego entre ambos, el paciente pueda asumir los estados disociados, y queden estos reconocidos e integrados en un self razonablemente cohesionado (Coderch, J., 2010, 2012).

La apertura a la realidad incide, notablemente, en el diálogo del psicoanálisis con el mundo de la ciencia, muy especialmente con las aportaciones de las neurociencias. Deseo, en este punto, lanzar un mensaje tranquilizador para alivio de los espíritus temerosos que piensan que atender a los avances de las neurociencias puede esclavizarnos a ellas. No ha de ser forzosamente así, sino que las formidables aportaciones de esas ciencias, a partir de la última década del pasado siglo, han proporcionado un empuje definitivo al psicoanálisis relacional que, desde Ferenzczi, Sullivan, Fromm, Frieda Fromm-Reichmann y otros avanzaba muy lenta y discretamente, casi a escondidas, ignorado por el psicoanálisis clásico, como una corriente soterrada de la que emergieron, como por sorpresa, espléndidos destellos de luz, tales como en Loewald, Balint, Farbairn y Winnicott, más adelante en Kohut y después en Mitchell, sólo para citar a los ya fallecidos. Y este avance ha sido posible y acelerado porque lo han propiciado los descubrimientos de las neurociencias, las cuales han sustentado empíricamente las hipótesis, conceptos, teorías y observaciones que forman la base del paradigma relacional, a la par que han refutado viejas creencias y anquilosados prejuicios. expresa. Este material es para uso científico y profesional exclusivamente y puede contener información clínica sensible. Los editores no se responsabilizan de los contenidos de los autores. Dirigir las consultas sobre derechos y autorizaciones a ceir@psicoterapiarelacional.es 
En mi Ponencia a la VII Reunión de IARPP-España (2017), hablé ampliamente (Coderch, J., 2015, 2016a, 2017) acerca de lo que denomino el genio terapéutico del psicoanálisis, así como de su vinculación con las más significativas contribuciones de las disciplinas confines en el fortalecimiento y extensión del campo del psicoanálisis, exposición que no voy a repetir ahora. Pero sí creo importante volver a recordar y seguir el hilo que inicié al referirme a la total corporalización de la mente, porque el cerebro se halla enteramente integrado al resto del organismo a través de circuitos neuronales y neuroquímicos, y en continua interacción con el medio que le rodea (Wagensberg, J. 1985; Sprueill, V., 1993; Damasio, A., 1999; Lakoff,G., Y Jonshon, M., 1999; Stolorow, R., 1997, 2011; Gallesse, V 2009; Gallese, V., Eagle, M, y Migone, O., 2007; Coburn, W, 2000,2002), y, así mismo, creo oportuno retomar la afirmación que entonces hice de que es a través de la sintonización psicobiológica de las mentes corporeizadas de paciente y analista que llega a producirse el verdadero cambio psíquico (2017p. 3-4).

Pues bien, creo que ahora son oportunas algunas reflexiones acerca de tal sintonización psicobiológica sustentada, en gran parte, por el sistema de neuronas en espejo (s.n.e.). El conocimiento de este proceso ha sido, en las dos últimas décadas, extraordinariamente ampliado merced a las aportaciones de grandes investigadores, entre ellos Gallesse, Eagle, Migone Rizzolatti y Sinigaglia, y esto nos ha permitido llegar a lo más hondo por lo que concierne a la esencia de las experiencias terapéuticas. Afirma Gallesse que los humanos podemos comprendernos los uno a los otros porque disponemos todos del mismo substrato neuronal que se activa cuando realizamos acciones o experimentamos emociones y sensaciones. $Y$ a este mecanismo neuronal funcional lo denomina simulación corporeizada (Gallesse, V., 2009; Gallesse, V., Eagle, M. y Migone, O., 2007). Gracias a esta función, no únicamente podemos comprender al otro cognitivamente, sino que lo reconocemos corporalmente, dentro de nuestro propio organismo. Ahora, por tanto, sabemos que cuando dos seres humanos interaccionan entre sí se activan los sistemas neuronales propios de sus pensamientos y de los sentimientos que están experimentando, de manera que se produce una profunda sintonización psicobiológica de lo que están sintiendo, con lo cual tiene lugar una forma directa de comprendernos los unos a los otros desde nuestro interior, puesto que se evocan en los interlocutores, recíprocamente, las representaciones internas de las sensaciones y los estados corporales asociados, como si cada uno de ellos estuviera experimentando emociones o sensaciones similares a las del otro (2009, p.41). Y, en un sentido muy semejante, se pronuncian G. Rizzolatti y G. Sinigaglia (2006), quienes nos hablan de un mecanismo espejo que permite a nuestro cerebro reconocer de inmediato todo lo que vemos sentimos o imaginamos que hacen y sienten los otros, gracias a la puesta en marcha de las mismas estructuras neurales (p.182). Por esto siempre se ha sabido que es muy difícil engañar a los niños. expresa. Este material es para uso científico y profesional exclusivamente y puede contener información clínica sensible. Los editores no se responsabilizan de los contenidos de los autores. Dirigir las consultas sobre derechos y autorizaciones a ceir@psicoterapiarelacional.es 
Señalan estos autores que el hecho de que las reacciones viscero-motoras no se expresen a nivel de los centros periféricos no implica irrelevancia, sino que se debe a que su función es la de representar tales reacciones para la comprensión interpersonal de las emociones ajenas, no el de ser necesariamente actuadas. Sin embargo, podemos matizar esta advertencia afirmando que, si bien la intensidad de la emoción simulada variará en función del impacto y de la historia de la persona que observa, los mecanismos de inhibición del comportamiento nos permiten ajustar la reacción a nuestra propia situación (Ginés, S., 1957).

Así las cosas, el descubrimiento de la función de la simulación corporeizada tiene consecuencias todavía no exploradas suficientemente para la comprensión de la formación de las sociedades humanas en todos sus aspectos, en el arte, la música, la comunicación, etc., campos que no forman parte de los objetivos de mi exposición, pero esta función sí es de extraordinario interés para los analistas en lo que concierne a la esencia de las experiencias que constituyen el genio terapéutico del psicoanálisis. Porque, mediante esta función, la comprensión empática del analista frente a sus pacientes puede ir mucho más lejos de la mera intelección consciente y cognitiva, merced al procesamiento paralelamente distribuido de la información recibida, del que nos habla el cognitivismo de la tercera generación, a través del cual todos aquellas señales sensoriales que los dos componentes de la díada analítica emiten ininterrumpidamente durante el diálogo (parpadeo, centelleo de los ojos, expresiones faciales, actitud y movimientos del cuerpo, mímica, gesticulación, etc.), imposibles de detectar en su gran totalidad por parte de la consciencia, pasan directamente a formar parte del inconsciente relacional, procedimental y no reprimido, configurando patrones o principios organizadores de respuesta, listos para intervenir en la mutua interacción cuando sean estimulados por determinadas circunstancias.

Para comprender mejor todo lo que acabo de decir, ha de quedar muy claro que el hecho de que en el cerebro del analista se activan las mismas zonas corticales y subcorticales que subyacen al estado mental del paciente, no significa que todo analista sienta siempre, verdaderamente, un interés personal, simpatía, compasión, afecto y deseos de ayudarle. Se trata de dos procesos distintos de los que el segundo implica el primero, pero no viceversa (Rizzolatti , G. y Sinigaglia, C., 2006). Yo creo que esto que acabo de decir es lo que permite comprender porque en todos los tratamientos psicoanalíticos y psicológicos de la más diversa índole, en los que hay uno, el paciente que pide ayuda, y otro, el profesional que intenta darla, hay éxitos y fracasos, por lo que ha resultado imposible, hasta el momento, demostrar, a nivel empírico, cuáles son la teoría de la mente y la metodología clínica más eficientes para el logro de la mejoría de los pacientes (Loewald, H., 1977; Wallerstein, R., 1989; Ávila-Espada, A, 2005, 2013, 2016a, 2016b; Guimón, J., 2007, 2014; Shedler, R., 2010, Coderch, J., 2010, 2012, 2015, 2016a, 2017) Y esto es así porque la eficacia del tratamiento psicoanalítico no depende de la 
orientación teórica y técnica del profesional, sino de su personalidad, es decir de su capacidad de sentir con el paciente, acompañarle en su sufrimiento, reconocerle en lo más íntimo de su ser y compartir sus sentimientos. De acuerdo con todo ello, pienso que ahora me es posible ampliar la anterior afirmación acerca del genio terapéutico del psicoanálisis, y para ello expreso mi conclusión de que el genio terapéutico de las experiencias que se dan en el psicoanálisis reside en el diálogo de implícito a implícito entre paciente y terapeuta, basado en la sintonización psicobiológica que promueve la activación simultánea de las mismas redes y estructuras en los cerebros de ambos.

Pienso, también, que todo lo que acabo de exponer en los últimos párrafos no debe extrañarnos, porque esto, simplemente, es lo que hacen las madres suficientemente buenas, de las que tan bien nos ha hablado Winnicott, en su diálogo con el bebé. Estas madres perciben en su interior, de forma inmediata, los estados mentales de su bebé y los reflejan en su rostro, tono y musicalidad de su voz, actitud y gestos, y el bebé, al mirar a la madre y también al padre si es el caso, internaliza de nuevo su estado mental, pero esta vez transformado en una representación secundaria o proto símbolo, cosa que permite le permite tomar una distancia respecto a estos estados mentales, no quedar invadido por ellos e ir progresivamente, de internalización a internalización, formando su capacidad de mentalización y su self. Esto mismo puede presentarse en el diálogo paciente- analista, cuando este último transmite al primero su afecto y su estar con él.

\section{El anhelo como el deseo que siempre es deseo.}

Siguiendo con las positivas consecuencias de la apertura del psicoanálisis a la realidad de la vida y al diálogo con otras disciplinas, creo que ello nos puede conducir al encuentro de otra categoría propia y exclusiva de los seres humanos a la que el psicoanálisis le ha prestado, hasta el momento, muy poca atención. No únicamente somos seres racionales, emocionales y simbólicos, sino que también somos seres anhelantes, movidos por la esperanza (Coderch, J., 2014). Esta categoría ha sido puesta de relieve por uno de los filósofos más importantes del pasado siglo, el filósofo abiertamente marxista, pero que hunde también sus raíces en el romanticismo y el expresionismo, Ernst Bloch, quien la desarrolló en su magna obra El Principio Esperanza, escrito entre 1938 y 1947, durante su exilio a los EE.UU., y luego reelaborado tras su retorno a la Alemania del Este. En esta extensísima obra, Bloch reflexiona sobre la naturaleza del ser humano, marcada por el anhelo, señalado por el deseo y la esperanza de lograr algo que siempre se percibe como más excelente, la necesidad de saciar un deseo que nunca desaparece: el deseo que siempre es deseo. 
Nos introduce Bloch en la naturaleza esencialmente deseosa, anhelante y trascendente de todos nosotros, hombres y mujeres, naturaleza que nos impulsa, más allá de satisfacciones físicas, de bienes materiales, de prestigio, poder y amor a encontrar algo que no podemos precisar exactamente lo que es, pero que sí sentimos que está siempre más allá, que nunca llegaremos a poseer totalmente, que es algo que nos trasciende; un anhelo, en definitiva, que es insaciable y que nunca descansa, en suma, como he dicho antes, el deseo que siempre es deseo porque nunca llegarán a coincidir las materializaciones históricas con aquello que ha de saciar el deseo estructural que anida en el ser humano. El deseo que no cesa forma parte inseparable de nuestra especie y la distingue, radical y definitivamente, del resto de los organismos vivos, como también nos separa de ellos el impulso a la trascendencia, es decir, a encontrar aquello que existe más allá de nuestros límites sensoriales y cognitivos, así como de nuestras experiencias. Bloch enlaza el anhelo con la consciencia utópica, o sea, la consciencia de traspasar los límites de lo que está normativamente establecido y sancionado. Según él, la esperanza irrumpe, como una revelación deslumbrante que rompe los moldes fijados, cuando el individuo percibe que son traspasables y que él más allá no lo será eternamente. Afirma Bloch:

La consciencia utópica quiere ver más allá ¿Cuál es el motor del anhelo? ¿Cuál es su fuerza motriz? ¿Quién impulsa en nosotros? Uno que se tiene a sí mismo, que todavía no surge. Ahora no hay más que decir, este interior dormita. La sangre corre, el corazón late sin que pueda percibirse que es lo que mantiene el pulso en movimiento ( $p .175$ del vol.1 de la ed. castellana).

Apunto que hay algo que es menester no olvidar por su enorme significado concerniente a lo más profundo de la intimidad de los humanos. Llevados por nuestra manera habitual de pensar, con facilidad podemos caer en el error de creer que el anhelo es lo que podemos considerar el primer movimiento, el cual llama en su auxilio a la esperanza. Y esto no es así, porque, como nos dice Bloch, lo primero es la esperanza, algo que brota de lo más íntimo de nuestro ser, como el primer motor, y de esta esperanza nace la fuerza para anhelar algo deseable que está más allá de nuestra contingencia y de nuestras limitaciones. Y ahora me atrevo a decir que tal vez, a partir de esta idea, podemos añadir una significativa matización a la definición del ser humano: un ser que, impulsado por la esperanza, anhela incansablemente algo que presiente como su verdadera patria.

Para Bloch la verdadera patria era la patria socialista. Dado que este término ha sido en política tan tergiversado y empleado con muy diversos significados, yo me atrevería a decir que la verdadera patria de los seres humanos con la que soñaba Bloch es la fraternidad universal, y aún más, la indisoluble unidad de todo el Universo, en la que ahora coincide la teoría cuántica con los místicos orientales (Capra, F., 1975). 
Pienso que es de suma importancia no olvidar el papel que juega la esperanza en la clínica psicoanalítica. Si el analista no se siente vinculado con aquel a quien está tratando de ayudar, y no alimenta en él mismo la esperanza de lograr que, con su esfuerzo, tengan lugar en los procesos psíquicos del paciente los cambios necesarios, será muy difícil que éste se sienta movido por la esperanza. Con relación a esto, es muy común en el mundo psicoanalítico el sentimiento de que los analistas jóvenes, pletóricos de ilusión y de entusiasmo, consiguen ayudar a pacientes en los que fracasan los más sabios y experimentados, pero también desengañados por muchas decepciones, viejos analistas (Berman, E., 2004).

El texto de Bloch rezuma, constantemente, la nostalgia de una utopía, la utopía socialista que creyó al alcance de la mano en un momento de la historia, y que luego vio fracasada. Pero la nostalgia de Bloch se muestra llena de la esperanza de que, finalmente, la humanidad llegará a su verdadera patria. Sin embargo, su esperanza no se limita al logro de la patria socialista, sino que, así mismo, se centra en el desarrollo del hombre, ser inconcluso, "utópico" por naturaleza, que precisa seguir desarrollándose para asumir su verdadero papel de protagonista de la historia. Dice así este filósofo:

Lo que se le representa hacia adelante al instinto de ampliación del yo es más bien, como ya tendremos ocasión de mostrar, algo que no ha existido ni ha sido consciente en el pasado, un "todavía-no consciente" y, por tanto, en sí, un alba hacia adelante, hacia lo nuevo. Éste es el amanecer que puede ya rodear los sueños diurnos más simples; de aquí llega a las zonas más amplias de la privación que se rechaza, es decir, de la esperanza (vol l, pp.107 de la ed, castellana; comillas del autor).

Por mi parte, pienso que esta caracterización del sujeto humano como un ser anhelante, hunde sus raíces en las bases metafísicas de Platón. Éste nos dice que la historia del hombre comienza antes de nacer, cuando todavía es un alma alada en el espacio. Las almas que posteriormente se encarnan en los humanos, a diferencia de las que caen en los animales, han gozado de la excelsa visión de las Ideas, el sumo Bien, la suma Justicia, la suma Belleza. Cuando se hallan en la tierra, en las más sensibles entre ellas surge una nostalgia invencible y el constante anhelo de volver a contemplar las Ideas. Así, a la vez en Bloch y en Platón podemos apoyarnos para sustentar el concepto del ente humano como no sólo racional y

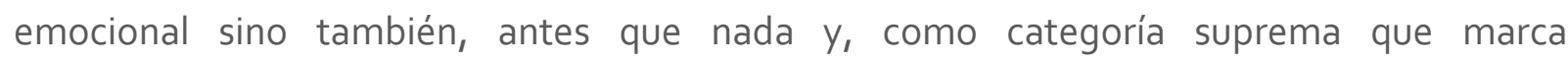
inconfundiblemente su exclusiva peculiaridad, como un ser que anhela, perennemente, trascenderse a sí mismo.

En el otro extremo del saber, la neurobiología, también nos habla hoy en día, de la importancia del anhelo como categoría humana que siempre hemos de tener muy presente. expresa. Este material es para uso científico y profesional exclusivamente y puede contener información clínica sensible. Los editores no se responsabilizan de los contenidos de los autores. Dirigir las consultas sobre derechos y autorizaciones a ceir@psicoterapiarelacional.es 
A. Damasio, preguntándose sobre la vinculación entre el anhelo y la trascendencia expresa (2006):

Se podría contestar afirmando que el anhelo es un rasgo profundo de la mente humana. Está implantado en el diseño del cerebro humano y en el acervo genético que lo engendra, no menos que en los rasgos profundos que nos conducen con gran curiosidad hacia una exploración sistemática de nuestro propio ser y del mundo que lo rodea: los mismos rasgos que nos impulsan a construir explicaciones para los objetos y situaciones de este mundo (p.248).

Yo pienso que las reflexiones que acabo de exponer nos impelen con fuerza a considerar el anhelo, como categoría suprema del ser humano, el verdadero motor de su ininterrumpida evolución.

Ya no puedo seguir adelante por este camino. Pero deseo añadir, antes de terminar con mis reflexiones acerca de las consecuencias que la apertura del psicoanálisis a la realidad ha tenido para sus propios fundamentos y para la comprensión de los seres humanos, que la historia parece dar la razón a Bloch en su sueño de la patria socialista como meta final de la humanidad. Digo esto porque los seres humanos siempre han sido dados a imaginar lo que Ilamamos utopías, termino tomado de la que escribió Tomas Moro, Canciller de Enrique VIII de Inglaterra por quien terminó siendo ejecutado: Vtopiae. Porque sucede que en las utopías los hombres y las mujeres imaginan una anhelada sociedad, pasada y que se perdió en la memoria de los tiempos, pero que ha de retornar, o futura, que está por venir, en la que reinarán la justicia, la igualdad, la paz y el bienestar para todos. En ella no habrá diferencias sociales ni económicas, ni, por lo que parece, luchas por el dinero y el poder. Es decir, creo que los humanos que, una y otra vez, tal situación, pretérita o pasada, imaginan nos retratan en sus utopías sociedades socialistas perfectas. Pero Bloch no encontró en la Alemania del Este la armonía y la libertad buscadas, porque siempre, inevitablemente, las utopías, huidizos y evanescentes sueños, se deshacen en nuestras manos bajo el soplo de la realidad y dejan de ser utopías cuando creemos encontrarlas, y Bloch se exilió de nuevo, esta vez a Suiza, pero no por esto desistió de su empeño, ni dejó de luchar con la pluma para que su particular utopía llegara a hacerse realidad, movido por la esperanza, sin duda.

\section{REFERENCIAS}

ÁVILA-ESPADA, A. (2005). Al cambio psíquico se accede por la relación, Intersubjetivo, vol.2, num. 1, pp.: 195- 220 .

ÁVILA-ESPADA, A. (2013). La relación contextual determinante de transformación. Reflexiones en torno al papel determinante del Insigth y de la experiencia de relación en el cambio psíquico, Temas de Psicoanálisis, num.6. 
ÁVILA-ESPADA, A.(ed.) (2014). La Tradición Interpersonal. Perspectiva Social y Cultural del Psicoanálisis, Madrid: Ágora-Relacional.

ÁVILA-ESPADA, A. (2015). Entrevista en Temas de Psicoanálisis, num.9.

ÁVILA-ESPADA, A. (2016a). Perspectivas relacionales frente al conflicto social: el compromiso del clínico relacional con la acción transformadora, Clínica e Investigación Relacional, vol.10 (3), pp. 723727.

ÁVILA-ESPADA, A. (2016b). La evolución de la clínica relacional: Tendencias y Retos. Conferencia de Apertura de las I Jornadas de Psicoanálisis Relacional, Salamanca.

BALADIER, G. [1988]. El Desorden, Barcelona: Gedisa, 2003.

BARCELLONA, P. (1992). Postmodernidad y Comunidad, Madrid: Ed. Trotta.

BAUMAN, Z. (2000). Modernidad Líquida, México: Fondo de Cultura Económica.

BAUMAN, Z. (2001). La Sociedad Individualizada, Madrid: Ediciones Cátedra.

BERMAN, E. (2004), Impossible Training, Londres: The Analytic Press.

BLOCH, E. [1959]. El Principio Esperanza, Madrid: Ed. Trotta, 2007.

BROMBERG, P. (2011). The Shadow of the Tsunami, and the Grow of the Relational Mind, Londres: Routledge.

CAPRA, F. [1975]. El Tao de la Física, Málaga: Ed. Sirio, 2017.

CASTAÑO, R. (2011). La Terapia Sexual. Una Mirada Relacional, Madrid: Ágora Relacional.

COBURN, W. (2000). The organizing forces of contemporary psichoanalysis: Reflections on nonlinear dynamics systems theory, Psychoanal.Psychol.,17:750-770.

COBURN, W. (2002). A World of systems: The role of the systematic patterns of experience in the therapeutic process, Psychoanal, Inq., 22: 665-667.

CODERCH, J. (2010). La Práctica de la Psicoterapia Relacional, Madrid: Ágora Relacional.

CODERCH, J. (2012). Realidad, Interacción y Cambio psíquico, Madrid: Ágora Relacional.

CODERCH, J. (2014). La puerta abierta, Clínica e Investigación Relacional, vol.8 (2), pp-: 359-373.

CODERCH, J. (2015). Las experiencias terapéuticas en psicoanálisis relacional desde la perspectiva de la no linealidad I, Temas de Psicoanálisis, num.9.

CODERCH, J. (2016a). Las experiencias terapéuticas en psicoanálisis relacional desde la perspectiva de la no linealidad II, Temas de Psicoanálisis, num.11.

CODERCH, J. (2016b). Comprendiendo a una sociedad en cambio para comprender a los pacientes, Clínica e Investigación Relacional, Clínica e Investigación Relacional, vol.11 (1), pp.51-69.

CODERCH, J. (2017). Las experiencias terapéuticas modifican el inconsciente relacional, Clínica a Investigación Relacional, vol.1 (2), pp.307.

CODERCH, J, CASTAÑO, R., CODOSERO, A., DAURELLA, N., y RODRÍGUEZ-SUTIL, C. (2014). Avances en Psicoanálisis Relacional, Madrid: Ágora Relacional. 
CODERCH, J. y PLAZA, A. (2016). Emoción y Relaciones Humanas, Madrid: Ágora Relacional.

CODOSERO, A. (2012). La evolución de la teoría traumática, en J.Coderch Realidad,Interacción y Cambio Psíquico, cap.2.

DAMASIO, A. (1999]. La Sensación de lo que Ocurre, Madrid: Debate. 2001.

DAMASIO, A. (2006). En busca de Spinoza, Barcelona: Ed. Crítica.

DUCH, L. y MĖLICH, J.C. (2004). Ambigüitats de l'Amor, Publicacions de l'Abadia de Montserrat.

FERENCZI, S. (1997). Sin Simpatía no hay Curación. El Diario Clínico de 1932, Buenos Aires: Amorrortu [Publicación original]-Dupont (Ed.), 1985,1988].

GALLESSE, V. ((2009). Neuronas en espejo, simulación corporeizada y las bases neurales de la identificación social, Clínica e Investigación Relacional, vol. 5 (19) pp: 34-50, 2011.

GALLESE, V., Eagle, M., y MIGONE, O (2007). Intentional attunement. Mirror neurons and neural underpinning of interpersonal relations, J. Amer. Psychoanal. Assn., 55:131.175 . .

GUERRA-Cid, R. (2013). El Clavo Ardiendo, Barcelona: Octaedro.

GUIMÓN, J. (2007).Crisis y Porvenir del Psicoanálisis, Bilbao: Universidad de Deusto.

GINÉS, S. (2017). Comunicación personal.

GUIMÓN, J. (2014). Globalización y Salud Mental, Madrid: Ed. Eneida.

GINÉS, S. (2017). Comunicación personal.

KANDEL, E., SCHWARTZ, J. y JESELL, T. (2001). Principios de Neurociencia, Madrid: Macgraw-Hill Interamericana.

KANDEL, E. [2005]. Psiquiatría, Psicoanálisis y la Nueva Biología de la Mente, Barcelona: Ars Médica, 2006.

LAKOFF, G. y JONSHON,M. (1999). Philosophy of the Flesh, Nueva York: Basic Books.

LIPOVETSKY,G. [2004]. Los Tiempos Hipermodernos, Barcelona: Anagrama.

LOEWALD, H. (1977). Primary process, secondary process and language, en Papers on Psychoanalysis, New Haven: Yale Univ. Press., pp. 178-2006.

LYOTARD,J.F. [1984]. La Condición Postmoderna, Madrid: Cátedra, 1994

MACINTYRE, A. [1984]. Tras la Virtud, Barcelona: Crítica.

ORANGE, D. (2006). For whom the bell tolls; context, complexity and compassion in psychoanalysis, Int, Psychoanal. Self Psycho., 1:5-21.

RIZZOLATI, G., y SINIGAGLIA, C. (2006). Las Neuronas Espejo, Barcelona: Paidós.

SENNET, R. [1998]. La Corrosión del Carácter, Barcelona: Anagrama, 2000.

SHEDLER, J. (2010). The efficacy of the psychodynamic psychotherapy, American Psychologist, 65: (2),98-109. 
SOUZA, M a de LOURDES (1999). La individualidad postmoderna. Una lectura del pensamiento de Pietro Barcellona y Bonventura da Sousa Santos, Archivos Electrónicos de Filosofía del Derecho, num. 2.

SPRUEILL, Van R. (1993). Determinist caos and the science of complexity: Psychoanalysis in the midst of general scientific revolution, J.Amer.Psychoanal. Assn.,41: 3-44.

STERN, D. (2004). The Present Moment, Nueva York.WWE.: Norton \&Company.

STOLOROW, R. (1997). Dyadic intersubjetive systems: An evolving paradigm for psychoanalysis, Psychoanal. Psychol., 14: 337-346.

STOLOROW, R, (2011) World, Affectivity, Trauma: Heidegger and Post Cartesian Psychoanalysis, EUA: Routledge.

SUMMERS, F. (2015). Implicaciones clínicas desde la perspectiva fenomenológica del psicoanálisis, Clínica e Investigación Relacional, vol.9 (1),pp:33-82.

TIZÓN, J. (2010). El Poder de la Por, Lleida: Pagès Editors.

TIZÓN, J. (2015). Psicopatología de la Corrupción, Barcelona: Herder.

TURNER, V. (1988). El Proceso Ritual. Estructura y Antiestructura, Madrid: Taurus.

WACHTEL, P.L. (2012). Reflections on the therapeutic process, Psychoanalytic Perspectives, 9: 88-117.

WAGENSBERG, J. [1985]. Ideas Sobre la Complejidad del Mundo, Barcelona: Tusquets, 2007.

WALLERSTEIN, R. (1989). Psychotherapy research Project of the Menninger Foundation, J. Consult. Clin. Psychol. 57: 157-205.

Original recibido con fecha: 25/01/2018 Revisado: 25/02/2016 Aceptado: 28/02/2018

NOTAS:

\begin{abstract}
${ }^{1}$ Trabajo leído como conferencia de clausura en las V Jornadas de Psicoanálisis Relacional celebradas por el Instituto de Psicoterapia Relacional en el Real Sitio de La Granja de San Ildefonso (Segovia, España) los días 23 y 24 de Febrero de 2018.

2 Joan Coderch de Sans (Hospitalet de Llobregat, Barcelona, 1930). Doctor en medicina y miembro titular de la Sociedad Española de Psicoanálisis, de la que ha sido presidente, y de la Asociación Psicoanalítica Internacional. Ex -Profesor Adjunto de Psiquiatría de la Universidad de Central Barcelona, Profesor Emérito de la Universidad Ramón Llull. Ha publicado los siguientes libros: Psiquiatría Dinámica (1975), Herder, segunda ed. modificada, 2012; Teoría y Técnica de la Psicoterapia Psicoanalítica (1987),Herder; La Interpretación en Psicoanálisis (1995), Herder; La Relación Paciente- Terapeuta (2001), Fundació Vidal i Barraquer-Paidós, (2012), Herder; Pluralidad y Diálogo en Psicoanálisis (2006), Herder; La Práctica de la Psicoterapia Relacional (2010), Madrid: Ágora Relacional; Realidad, Interacción y Cambio Psíquico (2012), Madrid: Ágora Relacional; Avances en Psicoanálisis Relacional (2014), Madrid: Ágora Relacional; Emoción y Relaciones Humanas (2016, con Alejandra Plaza), Madrid: Ágora Relacional. Contacto: 2897jcs@comb.cat

3 Los trabajos de todos estos autores se publican en este mismo número de CeIR.
\end{abstract} expresa. Este material es para uso científico y profesional exclusivamente y puede contener información clínica sensible. Los editores no se responsabilizan de los contenidos de los autores. Dirigir las consultas sobre derechos y autorizaciones a ceir@psicoterapiarelacional.es 